\title{
Comparison of sleep disturbance among patients with cancer and thalassaemia and healthy controls: a cross-sectional study
}

\author{
Salehoddin Bouya', Seyed-Mehdi Hashemi², Mohammadreza Hormozi ${ }^{3}$, Nezarali Moulae4, \\ Mahdi Mohammadi ${ }^{5}$, Afshin Imami ${ }^{3}$
}

\begin{abstract}
IInternal Medicine and Nephrology, Clinical Immunology Research Centre, Ali-Ebne Abitaleb Hospital, Zahedan University of Medical Sciences, Zahedan, Iran

${ }^{2}$ Clinical Immunology Research Centre, Department Of Internal Medicine, Haematology and Medical Oncology Ward, Ali-Ebne-Abitalelb Hospital, Zahedan University of Medical Science, Zahedan, Iran

${ }^{3}$ Department of Psychiatry, Zahedan University of Medical Science, Zahedan, Iran

${ }^{4}$ Pulmonary Diseases, Department of Internal Medicine, Ali IbneAbitaleb Hospital, Zahedan University of Medical Sciences, Zahedan, Iran

${ }^{5}$ Department of Biostatistics and Epidemiology, School of Health, Health Promotion Research Centre, Zahedan University of Medical Sciences, Zahedan, Iran
\end{abstract}

\section{Abstract}

Introduction: Sleep disturbance is an unwanted condition among healthy people and those with chronic diseases. This study was performed to compare sleep disturbance between cancer and thalassaemia major patients, and healthy controls.

Material and methods: This cross-sectional study was performed on 505 patients referred to 3 teaching hospitals in south-eastern Iran. Patients were selected through the convenience sampling method. Data were collected using the standard Pittsburgh Sleep Quality Questionnaire.

Results: The mean age of patients was 47.1 years (range 14-60 years). Most of the participants were female $(70 \%)$, city residents $(53 \%)$, and had a diploma $(47 \%)$. The overall prevalence of sleep disturbance in thalassaemia, cancer, and healthy controls was $91.9 \%, 98.2 \%$, and $94.7 \%$, respectively. The results also showed that there was no significant difference in the prevalence of sleep disturbance between thalassaemia and healthy controls $(p=0.27)$, but it was shown that people with cancer had significantly higher and more severe sleep disturbance compared to healthy controls $(p=0.01)$.

Conclusions: The high prevalence of sleep disturbance in thalassaemia and cancer patients indicates the importance of paying attention to the psychological dimension of patients along with the physical dimension, and designing appropriate training programs to improve sleep disturbance in patients.

Key words: cancer, thalassaemia major, sleep disturbance.

\section{Address for correspondence:}

Seyed-Mehdi Hashemi MD, Clinical Immunology Research Centre, Department of Internal Medicine, Haematology and Medical Oncology Ward, Ali-Ebne-Abitalelb Hospital, Zahedan University of Medical Science, Zahedan, Iran, e-mail: szmehdihashemi1990@gmail.com

\section{INTRODUCTION}

Sleep is one of the basic daily needs of human beings. Sleep promotes cellular repair, comfort, relaxation, and physical-mental rest [1]. Various studies have shown that sleep is impaired in healthy people and especially in patients [2,3]. Patients with chronic diseases suffer from various physical and mental disorders, including sleep disturbance [4]. Chronic diseases are always a major contributor to disability and mortality [5]. Studies have shown that there is a direct relationship between sleep disturbance and insomnia, and chronic diseases such as type 2 diabetes, heart disorders, obesity, and cancer [6]. Accord- ing to the latest statistics, by 2030, half of the population will suffer from at least one chronic disease [7]. Sleep disorders are major problems in chronic diseases such as cancer and thalassaemia [8]. Cancer is one of the most common problems worldwide. It is one of the 10 leading causes of death in the world, and despite tremendous progress in reducing the complications of the disease, these patients suffer from various complications, including sleep disturbance [9]. Studies have demonstrated that $30-75 \%$ of newly diagnosed cancer patients suffer from a variety of sleep disorders [10]. Sleep disturbance in these patients causes persistent fatigue, reduced adherence to treatment, and impaired quality of life [11]. 
Despite the importance of sleep quality, this disorder is often overlooked in patients, so that only $17 \%$ of patients with a sleep disorder are examined and treated by physicians [12]. Thalassaemia is a chronic inherited disorder and the most common genetic disease worldwide with a prevalence of about $3.6 \%$ [13]. This disease reduces the production of haemoglobin [13]. In Iran, there are over 20,000 patients with thalassaemia, to which more than 1500 people are added annually [14].

Thalassaemia major causes various physical and psychological complications in patients. One of the main concerns in these patients is a high level of anxiety, which causes sleep disturbance in them [15]. In Iran, few studies have been conducted in this field. Therefore, this study was performed to compare sleep disturbance among patients with cancer and thalassaemia major and healthy controls.

\section{MATERIAL AND METHODS}

This cross-sectional study was performed on 505 participants (187 healthy controls, 169 cancer patients, and 149 patients with thalassaemia major) referred to educational hospitals in south-eastern Iran from 1 March 2020, to 1 December 2020.

Patients were selected through the convenience sampling method. Inclusion criteria were age 1560 years, patients with known cancer or thalassaemia confirmed by a physician. Patients who had depressive disorders or restless legs syndrome simultaneously, with a history of drug use, and those who did not consent to participate in the study were excluded. The sample size was retrospectively selected using the information of patients referred to hospitals and clinics in the last year.

The standard Pittsburgh Sleep Quality Index (PSQI) was used [16]. This questionnaire consisted of 2 parts. The first part comprised demographic characteristics including age, gender, marital status, and level of education. The second part was the PSQI, which has 19 questions. The Likert scale was used to score the items. The dimensions of the questionnaire include subjective sleep quality, sleep latency, sleep duration, habitual sleep efficiency, sleep disturbances, use of sleeping medication, and daytime dysfunction. The overall score of each scale is between 0 and 3 , and the interpretation is no problem sleeping (0), mild sleep disturbance (1), moderate sleep disturbance (2), and severe sleep disturbance (3). The validity and reliability of this questionnaire have been confirmed in various foreign and domestic studies. The reliability and validity of the PSQI were approved by a Cronbach's $\alpha$ of 0.77 in Iran [17].

To collect data, after receiving a letter of approval for data collection from the university, it was coor- dinated with the desired hospitals. Then, with the help of the ward officials, a meeting was held with the possible participants to explain the study objectives. Then the sleep questionnaire was distributed among the participants and they were given $15 \mathrm{~min}$ utes to complete it. The questionnaires of illiterate patients were completed by the researcher. Only $15(2 \%)$ participants in the 3 groups were illiterate. Finally, they were collected after the determined time.

This study was approved by the Ethics Committee of Zahedan University of Medical Sciences under the ethics number IR.ZAUMS.REC.1398.90. Written and oral consent was received from all participants, who were assured that their information will remain confidential. The STROBE checklist was used to report the study. Descriptive statistical tests (mean, standard deviation, frequency, and percentage) and analytical tests $\left(\chi^{2}\right)$ were used to describe the demographic characteristics of the participants. SPSS Version 18.0 for Windows (SPSS Inc., Chicago, IL, USA) was used to analyse the data. A $p$-value less than 0.05 was considered significant.

\section{RESULTS}

Out of 510 participants, 505 completed the questionnaires (99\% response rate). The mean age of patients was 47.1 years (range 14-60 years). Most of the participants were female (70\%), city residents (53\%), and had a diploma (47\%). Regarding the prevalence of sleep disturbance in thalassaemia patients, the results showed that most participants $(n=98,65.8 \%)$ had mild sleep disturbance. In general, the prevalence of various types of sleep disturbance in thalassaemia patients was $91.9 \%$. In the case of cancer patients, the results showed that most participants $(n=85,50.3 \%)$ had moderate sleep disturbance. In general, the prevalence of various types of sleep disturbance in cancer patients was $98.2 \%$. Unlike cancer patients, in healthy controls the results showed that most of the participants $(n=131,70.1 \%)$ had low sleep disturbance. The prevalence of various types of sleep disturbance in healthy controls was $94.7 \%$ (Table 1).

The results showed that there was no significant difference in sleep disturbance between thalassaemia patients and healthy controls $(p=0.27)$. But patients with cancer had significantly higher and more severe sleep disturbance compared to healthy controls $(p=0.01)$.

\section{DISCUSSION}

The present study was performed to evaluate the frequency of sleep disturbance among 3 groups: healthy controls, cancer patients, and thalassaemia 
Table 1. Comparison of sleep disturbance among patients with cancer and thalassaemia and healthy controls $(n=505)$

\begin{tabular}{|c|c|c|c|c|c|c|}
\hline \multirow{2}{*}{$\begin{array}{l}\text { Rate of sleep } \\
\text { disturbance }\end{array}$} & \multicolumn{2}{|c|}{ Cancer } & \multicolumn{2}{|c|}{ Thalassaemia } & \multicolumn{2}{|c|}{ Healthy } \\
\hline & $n$ & $\%$ & $n$ & $\%$ & $n$ & $\%$ \\
\hline none & 3 & 1.8 & 12 & 8.1 & 10 & 5.3 \\
\hline low & 71 & 42 & 98 & 65.8 & 131 & 70.1 \\
\hline moderate & 85 & 50.3 & 39 & 26.2 & 43 & 23 \\
\hline severe & 10 & 5.9 & 0 & 0 & 3 & 1.6 \\
\hline total & 169 & 100 & 149 & 100 & 187 & 100 \\
\hline
\end{tabular}

patients. The results showed that cancer patients had moderate to severe sleep disturbance, but those with thalassaemia major and the healthy controls had mild sleep disturbance. The findings of the study showed that sleep disorders in cancer patients are higher than in healthy controls, while in patients with thalassaemia major it is not significantly different from healthy controls. Based on the findings, it can be said that sleep disturbance in cancer patients is higher than in those with thalassaemia major and healthy controls. These findings are in line with the results of previous studies [18-20].

Also, the findings of AMM Santoso (2019) showed that the rate of sleep disturbance in cancer patients undergoing chemotherapy is $29 \%$ [21]. In this regard, according to the findings, it can be said that sleep is one of the main and essential human needs, and lack of sleep or reduced quality of sleep may have irreparable effects on the body [22]. Disruption of the sleepwake cycle leads to the disruption of other physiological functions of the body such as loss of appetite, feeling tired, lack of concentration, exacerbation of diseases, and physical problems [23, 24].

Insomnia can negatively affect energy, mood, concentration, and overall health. Anxiety and stress caused by cancer as a life-threatening event, fear of death, and chemotherapy can cause stress and nocturnal awakenings, which to some extent cause sleep problems and poor sleep quality [25]. In this regard, pain and fatigue caused by cancer can aggravate the sleep problems of these patients. However, people with thalassaemia major experience a lower risk of death, resulting in fewer sleep problems. Illness and physical pain affect the quality and quantity of sleep [26].

Poor sleep may impair a person's feelings, thoughts, and motivation, as well as increase stress, pain, and delays in improving overall physical condition and health $[27,28]$. Moreover, concerning the obtained results, it can be said that cancer patients need to be hospitalized, which sometimes takes a long time and causes sleep disturbance and insomnia. Sometimes hospitalized patients seem to be asleep, but usually, their sleep does not rejuvenate and has poor quality $[29,30]$. The results show that the quality of sleep in patients hospitalized in different wards of hospitals is low, so that $46.5 \%$ of them had poor sleep quality [31].

Patients with thalassaemia major have more successful treatments than cancer patients, and this can reduce their anxiety, stress, depression, and other mental health problems, which in turn can improve their sleep performance. The most important limitations of this study were as follows: This study was descriptive, so specific methodological limitations of this type of study should be considered. Also, the number of participants in each group was limited, so care should be taken in generalizing the results to other groups.

\section{CONCLUSIONS}

According to the findings, it can be said that the proximity of death and panic and the resulting stress may lead to more sleep disturbance in patients with cancer than in healthy controls and in patients with thalassaemia major. Therefore, it is more important to pay attention to the psychological dimensions in chronic patients.

The authors declare no conflict of interest.

\section{REFERENCES}

1. Farasat S, Dorsch JJ, Pearce AK, et al. Sleep and delirium in older adults. Curr Sleep Med Rep 2020; 1-13.

2. Leger D, Stepnowsky C. The economic and societal burden of excessive daytime sleepiness in patients with obstructive sleep apnea. Sleep Med Rev 2020; 51: 101275.

3. Freeman D, Sheaves B, Waite F, et al. Sleep disturbance and psychiatric disorders. Lancet Psychiatry 2020; 7: 628-637.

4. Gontijo Guerra S, Berbiche D, Vasiliadis H-M. Changes in instrumental activities of daily living functioning associated with concurrent common mental disorders and physical multimorbidity in older adults. Disabil Rehabil 2020; 1-9.

5. Zhan Y, Liu Y, Liu H, et al. Factors associated with insomnia among Chinese front-line nurses fighting against COVID-19 in Wuhan: a cross-sectional survey. J Nurs Manag 2020; 28 : 1525-1535. 
6. Choi Y, Choi JW. Association of sleep disturbance with risk of cardiovascular disease and all-cause mortality in patients with new-onset type 2 diabetes: data from the Korean NHIS-HEALS. Cardiovasc Diabetol 2020; 19: 1-8.

7. Harrold EC, Idris AF, Keegan NM, et al. Prevalence of insomnia in an oncology patient population: an Irish tertiary referral center experience. J Natl Compr Canc Netw 2020; 18: 1623-1630.

8. Güneş S. Sleep problems in children and adolescents with $\beta$-thalassemia major. Cukurova Med J 2019; 44: 1267-1271.

9. Arbyn M, Weiderpass E, Bruni L, et al. Estimates of incidence and mortality of cervical cancer in 2018: a worldwide analysis. Lancet Glob Health 2020; 8: e191-e203.

10. Strollo SE, Fallon EA, Gapstur SM, et al. Cancer-related problems, sleep quality, and sleep disturbance among long-term cancer survivors at 9-years post diagnosis. Sleep Med 2020; 65: $177-185$.

11. Wu H-S, Davis J, Chen L. Bright light shows promise in improving sleep, depression, and quality of life in women with breast cancer during chemotherapy: findings of a pilot study. Chronobiol Int 2021; 1-11.

12. Liang $X$, Harris HR, Hendryx $M$, et al. Sleep characteristics and risk of ovarian cancer among postmenopausal women. Cancer Prev Res 2021; 14: 55-64.

13. Angastiniotis M, Petrou M, Loukopoulos D, et al. The Prevention of thalassemia revisited: a historical and ethical perspective by the thalassemia international federation. Hemoglobin 2021; 1-13.

14. Jaripour ME, Hayatigolkhatmi K, Iranmanesh V, et al. Prevalence of $\beta$-thalassemia mutations among Northeastern Iranian population and their impacts on hematological indices and application of prenatal diagnosis, a seven-years study. Mediterr J Hematol Infect Dis 2018; 10: e2018042.

15. Al-Hakeim HK, Najm AH, Al-Dujaili AH, et al. Major depression in children with transfusion-dependent thalassemia is strongly associated with the combined effects of blood transfusion rate, iron overload, and increased pro-inflammatory cytokines. Neurotox Res 2020; 38: 228-241.

16. Buysse DJ, Reynolds III CF, Monk TH, et al. Quantification of subjective sleep quality in healthy elderly men and women using the Pittsburgh Sleep Quality Index (PSQI). Sleep 1991; 14: 331-338.

17. Moghaddam JF, Nakhaee N, Sheibani V, et al. Reliability and validity of the Persian version of the Pittsburgh Sleep Quality Index (PSQI-P). Sleep Breath 2012; 16: 79-82.

18. Halle IH, Westgaard TK, Wahba A, et al. Trajectory of sleep disturbances in patients undergoing lung cancer surgery: a prospective study. Interact Cardiovasc Thorac Sur 2017; 25: 285-291.

19. Badiyepeymaiejahromi Z, Poorgholami F, Taghizadeganzadeh $\mathrm{M}$, et al. Investigate the relationship between sleep quality and some blood parameters in thalassemia major patients referred to Cooley's ward at Motahari Hospital in Jahrom, Iran Biosci Biotechnol Res Asia 2016; 13: 1239-1245.

20. Jeon MS, Agar MR, Koh E-S, et al. Barriers to managing sleep disturbance in people with malignant brain tumours and their caregivers: a qualitative analysis of healthcare professionals' perception. Support Care Cancer 2021. doi: 10.1007/s00520020-05970-4.

21. Santoso AM, Jansen F, de Vries R, et al. Prevalence of sleep disturbances among head and neck cancer patients: a systematic review and meta-analysis. Sleep Med Rev 2019; 47: 62-73.

22. Mercier J, Savard J, Bernard P. Exercise interventions to improve sleep in cancer patients: a systematic review and meta-analysis. Sleep Med Rev 2017; 36: 43-56.

23. Chen HM, Cheung DST, Lin YY, et al. Relationships of exercise timing with sleep, fatigue and rest-activity rhythms of lung cancer patients in Taiwan: an exploratory study. Eur J Cancer Care 2020; 29: e13233.
24. Kim S, Han J, Lee MY, et al. The experience of cancer related fatigue, exercise and exercise adherence among women breast cancer survivors: Insights from focus group interviews. J Clin Nurs 2020; 29: 758-769.

25. Wang X, Wang N, Zhong L, et al. Prognostic value of depression and anxiety on breast cancer recurrence and mortality: a systematic review and meta-analysis of 282,203 patients. Molecular Psychiatry 2020; 25: 3186-3197.

26. Dorgaleleh S, Barahouie A, Naghipoor K, et al. Transfusion related adverse effects on beta-thalassemia major and new therapeutic approaches: a review study. Intern J Pediatr 2020; 8: 11651-11661.

27. Tariq H, Weng Q, Garavan TN, et al. Another sleepless night: does a leader's poor sleep lead to subordinate's poor sleep? A spillover/crossover perspective. J Sleep Res 2020; 29: e12904.

28. Hamel A, Bastien C, Jacques C, et al. Sleep or play online poker?: gambling behaviors and tilt symptoms while sleep deprived. Front Psychiatry 2021; 11: 600092.

29. Kulpatcharapong S, Chewcharat P, Ruxrungtham K, et al. Sleep quality of hospitalized patients, contributing factors, and prevalence of associated disorders. Sleep Disord 2020; 2020: 8518396.

30. Jakobsen G, Engstrøm M, Thronæs M, et al. Sleep quality in hospitalized patients with advanced cancer: an observational study using self-reports of sleep and actigraphy. Support Care Cancer 2020; 28: 2015-2023.

31. Matsuda R, Kohno T, Kohsaka S, et al. Psychological disturbances and their association with sleep disturbances in patients admitted for cardiovascular diseases. PloS One 2021; 16: e0244484. 